\title{
Percepção dos estudantes de Medicina frente ao ensino de terminalidade
}

\author{
Perception of Medical students regarding the teaching of terminality \\ Percepción de estudiantes de Medicina sobre la enseñanza del estado terminal
}

Recebido: 08/03/2021 | Revisado: 16/03/2021 | Aceito: 21/03/2021 | Publicado: 29/03/2021

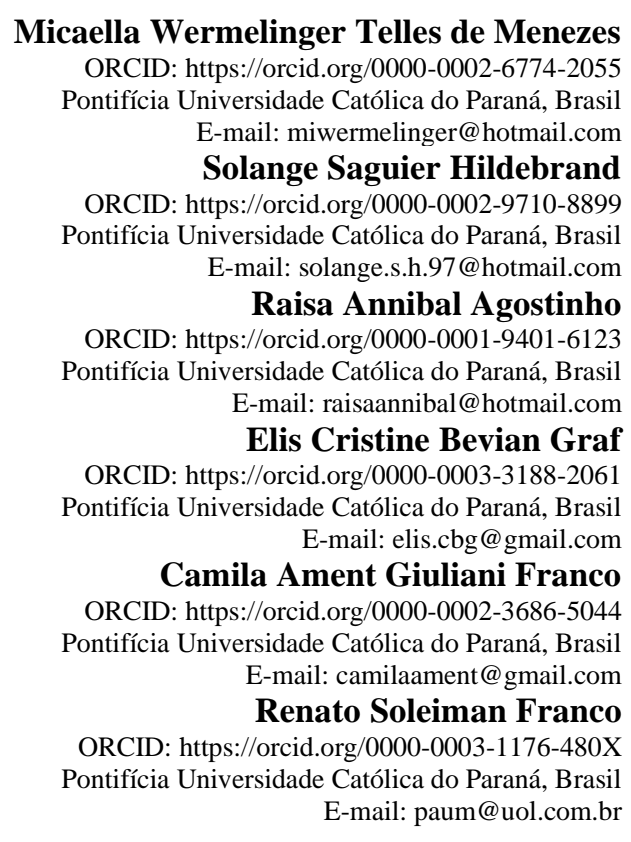

\section{Resumo}

O cuidado no fim da vida traz à tona a necessidade em conhecer como a terminalidade é encarada na formação médica, com especial atenção às atitudes dos estudantes. Com isso temos uma melhor compreensão dos fatores associados ao cuidar no contexto da terminalidade. Este estudo transversal avaliou a apreciação sobre o ensino de terminalidade de 347 estudantes das universidades de medicina de Curitiba-PR, sendo composta por, 72,8\% de mulheres e 43.8\% por acadêmicos no período clínico. A regressão linear mostrou que o gênero feminino ( $\beta$ :-0.290; $\mathrm{p}<0.0001)$ e a satisfação dos estudantes quanto ao seu currículo $(\beta:-0.111 ; p=0.03)$ são os principais fatores preditores de atitudes positivas frente a terminalidade. Assim, o papel das universidades em desenvolver currículos bem planejados e voltados para um ensino de qualidade é essencial. Por fim, a associação com gênero indica a possibilidade de diferentes modelos de cuidado estarem presentes e alguns deles mais alinhados a terminalidade.

Palavras-chave: Ensino; Educação médica; Estudantes de medicina; Assistência terminal; Terminalidade; Fim de vida.

\begin{abstract}
Care at the end of life brings out the need to know how terminality is seen in medical training, with special attention to students' behavoir. This gives us a better understanding of the factors associated with caring in the context of terminality. This cross-sectional study evaluated the assessment of the teaching of terminality of 347 students from the medical universities in Curitiba-PR, being composed by $72.8 \%$ of women and $43.8 \%$ of academics from the clinical period. Linear regression showed that female gender $(\beta:-0.290 ; p<0.0001)$ and student satisfaction with their curriculum $(\beta:-0.111 ; p=0.03)$ are the main predictors of positive attitudes towards terminality. Therefore, the role of universities in developing well-planned curricula focused on quality teaching is essential. Finally, the association with gender indicates the possibility of different models of care being present and some of them more aligned with terminality.
\end{abstract}

Keywords: Teaching; Medical education; Medical students; Terminal care; Terminal ill patients; End of life.

\section{Resumen}

El cuidado en fin de vida muestra la necesidad de saber cómo se ve el estado terminal en la formación médica, en especial las actitudes de los estudiantes. Esto da una mejor comprensión de los factores asociados con el cuidar en el contexto del estado terminal. Este estudio transversal evaluó la apreciación de la enseñanza del estado terminal a 347 estudiantes de las universidades médicas de Curitiba-PR, estando compuesta por el 72,8\% de mujeres y el $43,8 \%$ de 
académicos en el período clínico. La regresión lineal mostró que el género femenino $(\beta$ :-0,290; $<<0,0001)$ y la satisfacción de los estudiantes con su currículum $(\beta:-0,111 ; \mathrm{p}=0,03)$ son los principales predictores de actitudes positivas. Así, es esencial el papel de las universidades en la elaboración de planes de estudio bien planificados y centrados en calidad de enseñanza. Por último, la asociación con el género indica la posibilidad de que existan diferentes modelos de cuidado y algunos de ellos más alineados con el estado terminal.

Palabras clave: Enseñanza; Educación médica; Estudiantes de medicina; Asistencia en la terminal; Terminales; Fin de la vida.

\section{Introdução}

O cuidado a pacientes no fim da vida tem ganhado atenção na Educação Médica. Esse movimento voltado ao cuidar em situações em que curar não é mais possível talvez seja um resgate de uma das principais funções da Medicina, aliviar o sofrimento (Chaves et al, 2011). No entanto, a terminalidade coloca em choque modelos diferentes de cuidado como, por exemplo, o cuidado voltado às necessidades do sujeito e o voltado às expectativas do médico. A vontade curar e livrar o paciente da doença é mola propulsora para grande parte dos profissionais médicos. Evidente que curar é um desejo digno, mas na terminalidade essa função da prática profissional nem sempre é possível (Chaves et al, 2011; Pessini \& Hossne, 2010). Frente um paciente terminal, a compreensão e o alívio do sofrimento exigem uma atitude profissional centrada naquele que sofre e não na satisfação dos desejos (frequentemente de cura) dos profissionais. Uma vez que modelos centrado na doença (e no profissional) e nos pacientes coexistem na formação, é necessário conhecer como os estudantes tem percebido e vivenciado o ensino da terminalidade. Um melhor entendimento sobre a terminalidade na formação médica pode auxiliar na compreensão dos fatores mais associados a um cuidar digno na terminalidade e ao desenvolvimento dessas competências nos futuros profissionais.

A terminalidade de vida acontece quando se esgotam as possibilidades de resgate das condições de saúde e a possibilidade de morte próxima parece inevitável e previsível (Gutierrez, 2001). O objetivo é cuidar da família e do paciente para que esse momento possa ser o mais digno possível. A morte, muitas vezes evitada, passa a ser tema central e tem capital importância no estudo e entendimento da terminalidade (Collet \& Rozendo, 2003). Assim, um paciente terminal, pode não ter mais expectativas de cura da doença, mas ainda há muito a oferecer ao indivíduo (Marengo et al, 2009). Na realidade, uma vasta gama de ações pode ser tomada, tanto para o sujeito em questão, quanto para os que o rodeiam.

Oferecer os cuidados necessários na terminalidade requer uma boa comunicação, capacidade de avaliar desconforto emocional e preparação para atender questões psicológicas enfrentadas pelo enfermo (Barsansky et al, 1997). Desse modo, o cuidado na terminalidade baseia-se na empatia, comunicação e conhecimento técnico (Rosenthal \& Clay, 2017).

Mesmo com o conhecimento do que é necessário para o cuidado na terminalidade, o ensino médico é heterogêneo nesse sentido. Duas vertentes tendem a aparecer: a com foco primário e a integrativa (Toledo \& Priolli, 2012). A primeira é baseada em um modelo fragmentado entre as disciplinas, foca em conhecimento e conteúdos (Lampert, 2009). Já o modelo integrativo busca maior contextualização do aprendizado. Nele, a terminalidade é um tema que perpassa várias disciplinas e momentos de formação. Desse modo visa promover a importância do tema, o desenvolvimento de habilidade e atitudes em diferentes cenários de formação (Toledo \& Priolli, 2012; Campos et al, 2001).

Além da importância de buscar a melhor forma de ensino e aprendizado da terminalidade, é importante embasar a prática em teorias que sustentem o cuidado. Carol Gilligan (1982), destaca um Cuidado Ético voltado para a experiência da conexão com o outro, e uma maior importância na manutenção de relacionamentos de cuidado para a tomada das decisões (Erdmann, 1993). O Cuidado Ético é um modelo de "disposição relacional", voltado para uma interação dinâmica entre os sujeitos envolvidos no cuidado. Aliado a isso, considera as emoções e o contexto tanto dos profissionais como o de familiares e pacientes envolvidos nos processos de atenção (Kuhnen, 2014). Assim, as decisões e a atenção necessitam de uma atitude 
voltada ao cuidar do outro, com disponibilidade para uma conexão com empatia e alinhada com as emoções, necessidades e contexto do paciente.

O ensino da terminalidade exige a discussão de diferentes modelos do cuidar, um enfoque que vá além da cura de enfermidades e uma prática permeada por um ambiente de boa comunicação entre profissionais e pacientes/familiares (Fonseca \& Geovanini, 2013). Com isso, pode-se dizer que o ensino da terminalidade, frente às necessidades multivariadas é algo complexo. Desse modo, ao avaliar esse ensino sob o prisma do acadêmico de medicina, podemos conhecer não somente sua percepção sobre os métodos, como também, suas atitudes e valorização da terminalidade na formação.

\section{Metodologia}

$\mathrm{O}$ estudo caracteriza-se pelo delineamento observacional transversal, de natureza quantitativa como descrito por Pereira et al, de tipo survey e classificada como descritiva (Pereira et al, 2018). A pesquisa teve como base o questionário, elaborado pelos próprios pesquisadores, e intitulado a "Avaliação da apreciação dos estudantes de medicina sobre o ensino de terminalidade". O questionário é composto por afirmativas e as respostas graduadas em uma escala de Likert, sendo 1 concordo plenamente e 5 discordo plenamente.

As perguntas iniciais coletaram informações referentes à identificação do participante, como a instituição de ensino, período que cursava e gênero. As questões referentes a terminalidade foram desenvolvidas a fim de abranger: o que é ofertado na escola de medicina do participante; a importância do ensino de terminalidade (atitudes frente a terminalidade) e autoavaliação de competência.

A aplicação do questionário foi realizada por meio da plataforma eletrônica Qualtrics. Os participantes responderam ao questionário no próprio site da Qualtrics, por um link disponibilizado previamente. Este modelo de aplicação foi escolhido pela praticidade de coleta de dados e a facilidade de obter uma amostra maior.

Incluiu-se na amostra acadêmicos do curso de medicina de todos os períodos de cinco universidades de medicina do município de Curitiba - PR. O recrutamento dos participantes deu-se de três maneiras, sendo todas elas após a anuência do coordenador da instituição. A primeira através de contato por e-mail com os discentes da instituição, explicando a pesquisa e com um link para participação. A segunda através de envio de link por aplicativo de mensagem online, para os representantes de sala de cada instituição, solicitando a participação na pesquisa. Já a terceira forma com os pesquisadores visitando as salas com os discentes alvo da pesquisa, em dois momentos. O primeiro explicando a pesquisa para os alvos da entrevista e o segundo com a disponibilização do link para participação dos mesmos. Foram excluídos todos os participantes com menos de 18 anos de idade. A pesquisa foi avaliada e autorizada pelo CEP da Pontifícia Universidade Católica do Paraná, sob parecer número 3.024.840 de novembro de 2018.

\section{Análise dos dados}

As variáveis categóricas e numéricas foram descritas por meio de contagens (porcentagens) e médias (com respectivo desvio padrão), respectivamente. O questionário foi analisado por meio da análise de componentes principais. A dimensionalidade foi definida por um scree-plot, que foi utilizado para avaliar o número de componentes a ser considerado. Considerou-se que um item contribuía para um componente principal quando apresentava uma carga de valor superior a $|0,30|$ nesse componente. A consistência interna dos dados foi avaliada usando o alfa de Cronbach.

As associações lineares foram avaliadas usando coeficientes beta e intervalos de confiança de $95 \%$ do modelo de regressão linear múltipla. Os dados foram analisados por meio do Statistical Package for the Social Sciences (SPSS Versão 26). 


\section{Resultados}

\section{Resultados da Amostra}

Participaram da pesquisa 347 acadêmicos do curso de medicina das 5 universidades de Curitiba - PR (120, 34.6\% Universidade 1; 45, 13.0\% - Universidade 2; 75, 21.6\% - Universidade 3; 71, 20.5\% Universidade 4 e 36, $10.4 \%$ Universidade 5). A maior parte dos participantes eram mulheres, correspondendo a 72,8\% dos respondentes. Da amostra, $32.8 \%$ eram do pré-clínico ( $1^{\circ}$ ao $3^{\circ}$ período), $43.8 \%$ clínico ( $4^{\circ}$ ao $8^{\circ}$ período), e $23,4 \%$ do internato ( $9^{\circ}$ ao $12^{\circ}$ período). Somente 14 alunos eram menores de 18 anos e foram excluídos da análise.

\section{Análise do instrumento}

Os fatores com valor maior que $|0,30|$ agruparam as questões em um mesmo componente (grupamento) de questões, sendo que os 3 fatores foram responsáveis por uma variância explicada de 52.2\%. Assim, pudemos dividir o questionário em 3 componentes. O componente 1 incluiu 6 questões e foi intitulado "A análise do acadêmico sobre sua própria grade curricular" - chamaremos de Grade Curricular (GR) (alpha de Cronbach 0,82). Já o componente 2 agrupou 5 perguntas e foi intitulado “A opinião do acadêmico quanto a necessidade do ensino de terminalidade" - Atitude frente Ensino (AE) (alpha de Cronbach 0,68). O componente 3 apresentou um alpha de Cronbach baixo $(0,43)$ e desse modo os resultados não foram tratados como escore único, ou seja, não foi feita média entre os itens e cada um será apresentado individualmente (Quadro 1).

Quadro 1 - Formação dos componentes e as respectivas questões.

\begin{tabular}{|c|c|c|c|}
\hline \multirow[t]{2}{*}{ Análise de Componentes Principais } & \multicolumn{3}{|c|}{$\begin{array}{c}\begin{array}{c}\text { Componentes (Carga dos } \\
\text { Fatores) }\end{array} \\
\end{array}$} \\
\hline & 1 & 2 & 3 \\
\hline $\begin{array}{l}\text { Você acredita que a universidade na qual estuda proporciona embasamento } \\
\text { necessário para lidar com a terminalidade e a morte? }\end{array}$ & .676 & -.095 & .210 \\
\hline $\begin{array}{l}\text { A sua escola de Medicina foca no ensino de um atendimento voltado para a } \\
\text { unidade biopsicosocioespiritual do paciente? }\end{array}$ & .763 & .008 & .111 \\
\hline $\begin{array}{l}\text { Você acredita que durante sua formação acadêmica ensina-se a abranger o ser } \\
\text { humano de forma integrada e também sua doença? }\end{array}$ & .738 & -.029 & -.048 \\
\hline $\begin{array}{l}\text { Você acredita que durante a sua formação acadêmica recebeu informação } \\
\text { adequada para tratar sintomas mais comuns (dispneia, vômito, dor, diarreia e } \\
\text { caquexia) em pacientes terminais? }\end{array}$ & .731 & .034 & -.010 \\
\hline $\begin{array}{l}\text { Na sua graduação você recebeu preparo para atendimento completo de paciente } \\
\text { terminal (anamnese, exame físico e conduta)? }\end{array}$ & .722 & -.038 & .155 \\
\hline Você se sente confortável de como a morte é abordada durante a sua formação? & .638 & -.100 & .332 \\
\hline $\begin{array}{l}\text { Você considera importante abordar de forma mais efetiva o tema de morte e } \\
\text { terminalidade dentro do currículo acadêmico da sua escola de Medicina? }\end{array}$ & -.139 & .829 & -.009 \\
\hline $\begin{array}{l}\text { Você considera necessária uma disciplina inteiramente voltada para o ensino da } \\
\text { terminalidade? }\end{array}$ & -.167 & .702 & -.026 \\
\hline $\begin{array}{l}\text { Você considera aulas com o tema da terminalidade importantes para a formação } \\
\text { acadêmica? }\end{array}$ & -.067 & .784 & .032 \\
\hline Você acha importante o debate entre morte e morrer? & .037 & .600 & .096 \\
\hline $\begin{array}{l}\text { Você considera importante focar na unidade biopsicosocioespiritual durante o } \\
\text { atendimento do paciente? }\end{array}$ & .137 & .597 & -.120 \\
\hline $\begin{array}{l}\text { 3.1 Você sente-se confortável ao falar sobre morte (no sentido de finitude de } \\
\text { vida)? }\end{array}$ & .139 & .017 & .753 \\
\hline $\begin{array}{l}\text { 3.2 Você já reservou um tempo para pensar sobre a própria morte, sua posição } \\
\text { frente a ela e à vida? }\end{array}$ & .042 & .116 & .535 \\
\hline 3.3 Você considera-se apto a dar uma má notícia? & .193 & -.190 & .691 \\
\hline
\end{tabular}

Fonte: Autores: 
Considerando a Escala Likert as pontuações mais próximas de 1 (1-5), seriam as pontuações que apontam melhores condições ou atitudes mais positivas, e 5 apontaria para insatisfação ou atitudes mais negativas. A GR, contida nas 6 primeiras questões apontadas pelo quadro, apresentou média de 2.67 (DP 0.884). Desse resultado podemos vislumbrar que o estudante de medicina ainda considera defeituoso o seu ensino de terminalidade, necessitando ainda uma maior elucidação de como seria o melhor jeito de abordar o tema na visão dos estudantes. Já a AE aparece com uma média de 1.35 (DP 0.569), o que mostra a importância que os estudantes dão ao tema. Os dois componentes não deixam de conversar entre si: os alunos que ao mesmo tempo acham sua GC não suficiente acreditam na importância de um ensino centrado na terminalidade e suas ramificações, fato que ainda não é posto em prática com maestria na visão dos estudantes. Já em relação as questões sobre a auto avaliação de competências frente a terminalidade tiveram as seguintes médias: Você sente-se confortável ao falar sobre morte (no sentido de finitude de vida)?, média de 2.09 (DP 1.118); Você já reservou um tempo para pensar sobre a própria morte, sua posição frente a ela e à vida?, média de 1.21 (0.406) e Você considera-se apto a dar uma má notícia?, média de 2.91 (DP 1.238).

\section{Variáveis associadas aos componentes}

Considerando como variável dependente as atitudes dos estudantes sobre o ensino da Terminalidade a regressão linear múltipla mostrou que: a GC, a Universidade e o gênero são os principais fatores preditores das atitudes dos estudantes frente ao ensino (Quadro 2).

Quadro 2 - Variáveis preditoras das Atitudes dos Estudantes.

\begin{tabular}{|l|l|l|l|l|l|}
\hline \multicolumn{1}{|c|}{ Variáveis } & \multicolumn{1}{|c|}{ B } & \multicolumn{1}{c|}{$\begin{array}{c}\text { Erro } \\
\text { Padrão }\end{array}$} & \multicolumn{2}{c|}{$\begin{array}{c}\text { Intervalo de } \\
\text { Confiança - 95\% }\end{array}$} & Valor-p. \\
\hline & & & Lower & Upper & \\
\hline Gênero - Feminino & -.290 & .0711 & -.429 & -.151 & $.000^{*}$ \\
\hline Gênero - Masculino & $0 \mathrm{a}$ &. &. &. &. \\
\hline Universidade 1 & .097 & .1080 & -.114 & .309 & .368 \\
\hline Universidade 2 & .170 & .1292 & -.083 & .423 & .188 \\
\hline Universidade 3 & .354 & .1133 & .132 & .576 & .002 \\
\hline Universidade 4 & .175 & .1234 & -.067 & .417 & .156 \\
\hline Universidade 5 & $0 \mathrm{a}$ &. &. &. &. \\
\hline Grade Curricular & -.111 & .0373 & -.184 & -.038 & .003 \\
\hline Período do Curso & .020 & .0110 & -.001 & .042 & .064 \\
\hline $\begin{array}{l}\text { Variável dependente: Atitude dos } \\
\text { Estudantes }\end{array}$ & \multicolumn{7}{|l}{} & & & \\
\hline p foi considerado significativo quando $<0.05 . *: \mathrm{p}<0.0001$ & & & \\
\hline
\end{tabular}

Fonte: Autores.

Considerando 0a como a variável de comparação utilizada no quadro, cabe ressaltar que a maior diferença encontrada na pesquisa como preditor da importância dada ao ensino da terminalidade foi o gênero do aluno. Tendo como base as alunas mulheres, temos uma diferença de -0.290 em relação ao público masculino questionado, tendo um p<0.0001 nessa análise. Quanto as universidades, podemos também ressaltar uma grande diferença comparando a Universidade 1, tendo sido a base para o 0a, e a Universidade 3, com +.354 , tendo um $\mathrm{p}=0,002$. Esse dado escancara as diferenças ensino e de satisfação dos alunos entre universidades dentro de uma mesma cidade, sendo de suma importância estudos futuros sobre a unificação os 
métodos de ensino eficazes para abordar o tema. Corroborando essa ideia, podemos ver como a grade curricular (GC) foi fator relevante nos resultados, com um $\mathrm{p}=0,003$.

\section{Discussão}

Os profissionais de saúde têm dificuldades em lidar com situações de vulnerabilidade, como finitude e fim de vida, sentimento de impotência, angústia e insatisfação (Souza \& Tavares, 2020). Apesar de haver poucos dados em âmbito nacional para vislumbrar o real interesse dado pelos alunos no aprendizado dos cuidados de fim de vida (Toledo \& Priolli, 2012), nosso estudo mostrou uma alta valoração da importância desse ensino. Não obstante, o presente estudo demostrou que a maneira em que o acadêmico julga o seu currículo, a universidade que estuda e o gênero tem um papel importante nas atitudes frente importância da terminalidade. Quanto mais positiva a atitude, mais o estudante julga ser insuficiente o seu currículo. Essa menor satisfação pode fazer com que busque alternativas de complementação, cursos de extensão ou até mesmo estudos próprios. Por outro lado, estudantes com menos atitudes positivas julgam o ensino mais adequado. Assim, quem precisaria mais (atitudes menos positivas) exige menos. Desse modo, é essencial que as escolas médicas se mobilizem para promover uma aprendizagem suficiente, podendo-se dar através de incentivos. Como sugere Souza \& Tavares, "desenvolvimento de habilidades de escuta sensível e qualificada, além de expressão não verbal, observação e acolhimento diante das reais necessidades do sujeito" (Souza \& Tavares, 2020).

Toledo e Priolli realizaram um estudo que inclui 179 escolas de medicina do Brasil. Foi demostrado que 40,4\% dos coordenadores desses cursos avaliaram como pequena a prioridade dada ao ensino de cuidados de fim de vida. Além disso, 73,7\% afirmaram que, apesar do estímulo tanto por coordenadores, quanto professores, o tempo despendido nesse ensino é insuficiente (Toledo \& Priolli, 2012). O ensino transversal, integrando diversas disciplinas na discussão da terminalidade tem aumentado e está presente em algumas instituições. No entanto, essa integração pode ocorrer de maneira insuficiente (Toledo \& Priolli, 2012; Toledo et al, 2010; Reich, 1995). Assegurar locais de prática, professores qualificados e um modelo de atenção voltado para as necessidades do paciente e familiares integrados no currículo são grandes desafios. Uma vez que o currículo e a universidade têm impacto sobre a atitude dos estudantes frente a importância da terminalidade, a responsabilidade dos cursos em aumentar e melhorar a promoção de atividades que estimulem esse aprendizado é fundamental. Outro ponto associado às atitudes dos estudantes frente a terminalidade é o gênero.

Em 1982, Carol Gilligan, autora de In a Different Voice: Psychological Theory and Women's Development, traz a ideia de duas perspectivas diferentes para a compreensão moral: uma "masculina", e outra "feminina" (Erdmann, 1993). A voz masculina parte de um princípio de padrão da moralidade, baseadas na justiça, respeito a direitos individuais e normais universais. Já a voz feminina, denominada a "diferente", é baseada na conexão com o outro, e a prioridade é atribuída à manutenção dos relacionamentos de cuidado na tomada de decisões morais (Erdmann, 1993). Gilligan chama tais ideias respectivamente como "ética da justiça" e "ética do cuidado/cuidado ético" (Toledo \& Priolli, 2012).

O Cuidado Ético de Gilligan está muito ligado ao cuidado na terminalidade por compartilharem a essência da conexão entre as pessoas, o reconhecimento da responsabilidade de uns pelos outros e da comunicação entre os envolvidos como fundamentos para um cuidado adequado (Toledo \& Priolli, 2012; Erdmann, 1993). A perspectiva do Cuidado Ético não tem como objetivo reforçar estereótipos ou propor um modelo masculino versus feminino, mas destaca que há diferentes formas de considerar o cuidado. Sua proposta seria colocar o cuidado como o bem principal, levando em conta a singularidade e a particularidade de cada situação, assim como considerar a vulnerabilidade e emoções como importantes fontes do conhecimento moral.

A importância do gênero feminino nas atitudes positivas reforça as características apontadas por Gilligan e ao mesmo tempo alerta para o desafio de como discutir o cuidado considerando as possíveis particularidades entre gênero. Discussão 
essa, que tem como objetivo compreender essas particularidades e melhorar o cuidado e não promover estigmas. Se por um lado os currículos têm sido insuficientes, a complexidade desse ensino, incluindo a consideração de diferentes perspectivas de cuidado por parte de estudantes, eleva ainda mais a dificuldade do desafio em promover uma aprendizagem de qualidade voltada para terminalidade.

\section{Limitações do estudo}

A amostra se compôs de forma aleatória, houve uma taxa de participação menor de estudantes dos últimos anos do curso, quando comparada ao número de participantes dos primeiros anos. Notou-se, também, uma predominância de certas escolas de medicina em relação à outras. Assim, os achados desse estudo devem ser considerados sob a luz de suas limitações.

\section{Conclusão}

As atitudes dos estudantes frente à terminalidade são influenciadas não só pelo contexto do ensino (universidade e currículo), como também, por elementos pessoais, como o gênero. Assim, as instituições têm importante papel em aperfeiçoar, se não criar, modelos de aprendizado que contemplem as particularidades individuais, assim como os avanços nos métodos para aprendizagem. Apesar do interesse crescente que tanto os estudantes quanto as instituições vêm atribuindo a terminalidade, ainda há deficiências no ensino que precisam ser corrigidas. É importante considerar pontos como: 1) aspectos pessoais influenciam nas atitudes dos estudantes, desse modo é imprescindível considerar estratégias para motivar, estimular, manter o interesse e o aprendizado considerando estudantes com diferentes pontos de vista e concepções de cuidado; 2) estudantes que precisam mais podem exigir menos, assim, cabe a universidade um papel fundamental em propor estratégias suficientes de ensino. Cabe ressaltar que, ainda que o interesse sobre o assunto esteja em franca ascensão, faltam estudos para elucidar certas questões. É interessante buscar respostas mais aprofundadas sobre a diferença de interesse e apreciação entre gêneros que foi encontrada no estudo, procurando e medindo formas para neutralizar essa assimetria. Além disso, é importante a realização de estudos visando especificamente o aperfeiçoamento dos métodos de ensino, buscando a eficácia de cada um para um melhor aprendizado ao final, a fim de diminuir a frequente falta de interesse ligada a grade curricular. Por último, ter um estudo direcionado especificamente ao último estágio da faculdade de medicina, o internato, seria de suma importância para entendermos o quanto a vivência prática nos estágios pode influenciar a visão dos estudantes de medicina em relação ao tema. A ensino da terminalidade é multifacetado, e tem tido sua importância reconhecida pelos estudantes, no entanto, destacamos que exige da universidade um papel central na melhoria da formação.

\section{Referências}

Barzansky, B., Veloski, J. J., Miller, R., \& Jonas, H. S. (1997). Education in end-of-life care during medical school and residency training. Academic Medicine, 74(10), 102-104.

Campos, F. E. de, Ferreira, J. R., Feuerwerker, L., Sena, R. R. de, Campos, J. J. B., Cordeiro, H., \& Jr., L. C. (2001). Caminhos para Aproximar a Formação de Profissionais de Saúde das Necessidades da Atenção Básica. Revista Brasileira de Educação Médica, 25(2), 53-59.

Chaves, J. H. B., Mendonça, V. L. G. de, Pessini, L., Rego, G., \& Nunes, R. (2011). Cuidados paliativos na prática médica: contexto bioético. Revista Dor, $12(3), 250-255$.

Collet, N., \& Rozendo, C. A. (2003). Humanização e trabalho na enfermagem. Revista Brasileira de Enfermagem, 56(2), $189-192$.

Conselho Nacional de Educação - Câmara de Educação Superior. (2001). Resolução CNE/CES n 4 , de 7 de novembro de 2001 - Diretrizes Curriculares Nacionais do Curso de Graduação em Medicina. Diário Oficial Da União, 4, 1-6. https://doi.org/10.1017/CBO9781107415324.004

Erdmann, A. L. (1993). Tendências dos sistemas organizacionais de enfermagem hospitalar: algumas contribuições. UFSC.

Fonseca, A., \& Geovanini, F. (2013). Cuidados paliativos na formação do profissional da área de saúde. Revista Brasileira de Educação Médica, 37(1), 120125. https://doi.org/10.1590/s0100-55022013000100017

Gutierrez, P. L. (2001). O que é o paciente terminal. Revista Da Associação Médica Brasileira, 47(2), 85-109. 
Research, Society and Development, v. 10, n. 4, e2510413679, 2021

(CC BY 4.0) | ISSN 2525-3409 | DOI: http://dx.doi.org/10.33448/rsd-v10i4.13679

Kuhnen, T. A. (2014). A ética do cuidado como teoria feminista (pp. 1-9). Universidade Estadual de Londrina.

Lampert, J. B. (2009). Tendências de mudanças na formação médica no Brasil: tipologia das escolas. Hucitec. (2th ed.).

Marengo, M. O., Flávio, D. A., \& Silva, R. H. A. da. (2009). Terminalidade de vida: bioética e humanização em saúde. Medicina (Riberão Preto), 42(3), 350357.

Pereira, A. S., Shitsuka, D. M., Parreira, F. J., \& Shitsuka, R. (2018). Metodologia da pesquisa científica. Núcleo de Tecnologia Educacional da Universidade Federal de Santa Maria para os cursos da UAB. https://repositorio.ufsm.br/bitstream/handle/1/15824/Lic_Computacao_Metodologia-PesquisaCientifica.pdf?sequence $=1$

Pessini, L., \& Hossne, W. S. (2010). Terminalidade da vida e o novo Código de Ética Médica. Revista Bioethikos, 4(2), 127-129.

Reich, W. T. (1995). History of the notion of care. Bioethics encyclopedia (2nd ed.). Macmillan Library.

Rosenthal, M. S., \& Clay, M. (2017). Initiatives for responding to medical trainees' moral distress about end-of-life cases. AMA Journal of Ethics, 19(6), 585594. https://doi.org/10.1001/journalofethics.2017.19.6.stas1-1706

Souza, O. A. B., \& Tavares, C. M. M. (2020). Humanização do processo de cuidar em enfermagem à pacientes em terminalidade da vida: não temos tempo a perder. Research, Society and Development, 9(8), http://dx.doi.org/10.33448/rsd-v9i8.5572

Toledo, A P, Martinello, L. Z., \& Priolli, D. G. (2010). Disciplina com foco primário em terminalidade da vida: sua obrigatoriedade é válida? (p. 131). Instituto de Ensino e Pesquisa do Hospital Sírio Libanês São Paulo.

Toledo, Andréia Padilha de, \& Priolli, D. G. (2012). Cuidados no fim da vida: o ensino médico no Brasil. Revista Brasileira de Educação Médica, 36(1), 109117. https://doi.org/10.1590/s0100-55022012000100015 